Article

\title{
On the Asymptotic Behavior of Advanced Differential Equations with a Non-Canonical Operator
}

\author{
Omar Bazighifan ${ }^{1,2,+} \mathbb{D}$ and Ioannis Dassios ${ }^{3, *,+}$ \\ 1 Department of Mathematics, Faculty of Science, Hadhramout University, Hadhramout 50512, Yemen; \\ o.bazighifan@gmail.com \\ 2 Department of Mathematics, Faculty of Education, Seiyun University, Hadhramout 50512, Yemen \\ 3 AMPSAS, University College Dublin, Dublin 4, Ireland \\ * Correspondence: ioannis.dassios@ucd.ie \\ $\dagger$ These authors contributed equally to this work.
}

Received: 16 April 2020; Accepted: 27 April 2020; Published: 30 April 2020

Abstract: In this paper, we aim to study the oscillatory behavior of a class of even-order advanced differential equations with a non-canonical operator. In addition, we present results on the asymptotic behavior of this type of equations and provide an example that illustrates our main results.

Keywords: oscillation; even-order; advanced differential equations; asymptotic behavior

\section{Introduction}

In recent decades, many authors have studied problems of a number of different classes of advanced differential equations including the asymptotic and oscillatory behavior of their solutions, see [1-8] and the references cited therein. For some more recent oscillation results, see [9-20]. The interest in studying advanced differential equations is also caused by the fact that they appear in models of several areas in science. In [21-23], singular systems of differential equations are used to study the dynamics and stability properties of electrical power systems. Some additional mathematical background on this can be found in [24]. Systems of differential equations with delays are used to study additional properties of electrical power systems in $[25,26]$. Non-linear advanced differential equations can be used to describe complex dynamical networks, see [27-29], and bring new insight to their stability. Furthermore, this type of equations can be also used in the modeling of dynamical networks of interacting free-bodies, see [30]. Finally, properties of advanced differential equations are used in the study of singular differential equations of fractional order, see [31,32]. Several other examples in Physics can be found in [33]. In this paper, we consider an even-order non-linear advanced differential equation with a non-canonical operator of the following type:

$$
L_{y}+q(v) g(y(\eta(v)))=0, \quad L_{y}:=\left(a(v)\left(y^{(\kappa-1)}(v)\right)^{\beta}\right)^{\prime},
$$

where $v \geq v_{0}, \kappa$ is even and $\beta$ is a quotient of odd positive integers. The operator $L_{y}$ is said to be in canonical form if $\int_{v_{0}}^{\infty} a^{-1 / \beta}(s) \mathrm{d} s=\infty$; otherwise, it is called noncanonical. Throughout this work, we suppose that:

C1: $a \in C^{1}\left(\left[v_{0}, \infty\right), \mathbb{R}\right), a(v)>0, a^{\prime}(v) \geq 0$,

C2: $q, \eta \in C\left(\left[v_{0}, \infty\right), \mathbb{R}\right), q(v) \geq 0, \eta(v) \geq v, \lim _{v \rightarrow \infty} \eta(v)=\infty$,

C3: $g \in C(\mathbb{R}, \mathbb{R})$ such that $g(x) / x^{\beta} \geq k>0$, for $x \neq 0$ and under the condition

$$
\zeta(v)=\int_{v_{0}}^{\infty} \frac{1}{a^{1 / \beta}(s)} \mathrm{d} s<\infty .
$$


Definition 1. The function $y \in C^{\kappa-1}\left[v_{y}, \infty\right), v_{y} \geq v_{0}$, is called a solution of (1), if $\left(y^{(\kappa-1)}(v)\right)^{\beta} \in$ $C^{1}\left[v_{y}, \infty\right)$, for $a \in C^{1}\left(\left[v_{0}, \infty\right), \mathbb{R}\right), a(v)>0$ and $y(v)$ satisfies (1) on $\left[v_{y}, \infty\right)$.

Definition 2. Let

$$
D=\left\{(v, s) \in \mathbb{R}^{2}: v \geq s \geq v_{0}\right\} \text { and } D_{0}=\left\{(v, s) \in \mathbb{R}^{2}: v>s \geq v_{0}\right\} .
$$

A kernel function $H_{i} \in C(D, \mathbb{R})$ is said to belong to the function class $\Im$, written by $H \in \Im$, if, for $i=1,2$,

(i) $H_{i}(v, s)>0$, on $D_{0}$ and $H_{i}(v, s)=0$ for $v \geq v_{0}$ with $(v, s) \notin D_{0}$;

(ii) $H_{i}(v, s)$ has a continuous and nonpositive partial derivative $\partial H_{i} / \partial s$ on $D_{0}$ and there exist functions $\tau, \vartheta \in C^{1}\left(\left[v_{0}, \infty\right),(0, \infty)\right)$ and $h_{i} \in C\left(D_{0}, \mathbb{R}\right)$ such that

$$
\frac{\partial}{\partial s} H_{1}(v, s)+\frac{\tau^{\prime}(s)}{\tau(s)} H_{1}(v, s)=h_{1}(v, s) H_{1}^{\beta /(\beta+1)}(v, s)
$$

and

$$
\frac{\partial}{\partial s} H_{2}(v, s)+\frac{\vartheta^{\prime}(s)}{\vartheta(s)} H_{2}(v, s)=h_{2}(v, s) \sqrt{H_{2}(v, s)} .
$$

Next we will discuss the results in [34-36]. Actually, our purpose in this article is to complement and improve these results. Agarwal et al. in [34,35] studied the even-order nonlinear advanced differential equations

$$
\left(\left(y^{(\kappa-1)}(v)\right)^{\beta}\right)^{\prime}+q(v) y^{\beta}(\eta(v))=0 .
$$

By means of the Riccati transformation technique, the authors established some oscillation criteria of (5). Grace and Lalli [36] investigated the second-order neutral Emden-Fowler delay dynamic equations

$$
y^{(\kappa)}(v)+q(v) y(\eta(v))=0,
$$

and established some new oscillation for (5) under the condition

$$
\int_{v_{0}}^{\infty} \frac{1}{a^{1 / \beta}(s)} \mathrm{d} s=\infty
$$

To prove this, we apply the previous results to the equation

$$
y^{(\kappa)}(v)+\frac{q_{0}}{v^{\kappa}} y(\lambda v)=0, v \geq 1
$$

if we set $\kappa=4$ and $\lambda=2$, then by applying conditions in [34-36] on Equation (8), we find the results in [35] improves those in [36]. Moreover, the those in [34] improves results in [35,36]. Thus, the motivation in our paper is to complement and improve results in [34-36]. We will use the following methods:

- Integral averaging technique.

- Riccati transformations technique.

- Method of comparison with second-order differential equations.

We will also use the following lemmas from (1):

Lemma 1 ([3]). If $y^{(i)}(v)>0, i=0,1, \ldots, \kappa$, and $y^{(\kappa+1)}(v)<0$, then

$$
\frac{y(v)}{v^{\kappa} / \kappa !} \geq \frac{y^{\prime}(v)}{v^{\kappa-1} /(\kappa-1) !} .
$$


Lemma 2 ([19]). Suppose that $y \in C^{\kappa}\left(\left[v_{0}, \infty\right),(0, \infty)\right), y^{(\kappa)}$ is of a fixed sign on $\left[v_{0}, \infty\right), y^{(\kappa)}$ not identically zero and there exists a $v_{1} \geq v_{0}$ such that

$$
y^{(\kappa-1)}(v) y^{(\kappa)}(v) \leq 0
$$

for all $v \geq v_{1}$. If we have $\lim _{v \rightarrow \infty} y(v) \neq 0$, then there exists $v_{\theta} \geq v_{1}$ such that

$$
y(v) \geq \frac{\theta}{(\kappa-1) !} v^{\kappa-1}\left|y^{(\kappa-1)}(v)\right|
$$

for every $\theta \in(0,1)$ and $v \geq v_{\theta}$.

Lemma 3 ([2]). Let $\beta$ be a ratio of two odd numbers, $V>0$ and $U$ are constants. Then

$$
U x-V x^{(\beta+1) / \beta} \leq \frac{\beta^{\beta}}{(\beta+1)^{\beta+1}} \frac{U^{\beta+1}}{V^{\beta}}, V>0 .
$$

Lemma 4. Suppose that $y$ is an eventually positive solution of (1). Then, there exist three possible cases:

$$
\begin{aligned}
\left(\mathbf{S}_{1}\right) & y(v)>0, y^{\prime}(v)>0, y^{\prime \prime}(v)>0, y^{(\kappa-1)}(v)>0, y^{(\kappa)}(v)<0 \\
\left(\mathbf{S}_{2}\right) & y(v)>0, y^{(r)}(v)>0, y^{(r+1)}(v)<0 \text { for all odd integer } \\
& r \in\{1,3, \ldots, \kappa-3\}, y^{(\kappa-1)}(v)>0, y^{(\kappa)}(v)<0 \\
\left(\mathbf{S}_{3}\right) \quad & y(v)>0, y^{(\kappa-2)}(v)>0, y^{(\kappa-1)}(v)<0, \quad L_{y} \leq 0
\end{aligned}
$$

for $v \geq v_{1}$, where $v_{1} \geq v_{0}$ is sufficiently large.

\section{Oscillation Criteria}

Theorem 1. Assume that (2) holds. If the differential equations

$$
\begin{gathered}
\left(\frac{(\kappa-2) ! a^{\frac{1}{\beta}}(v)}{\left(\theta v^{\kappa-2}\right)^{\beta}}\left(y^{\prime}(v)\right)^{\beta}\right)^{\prime}+k q(v) y^{\beta}(v)=0, \quad \forall \theta \in(0,1), \\
y^{\prime \prime}(v)+y(v) \frac{1}{(\kappa-4) !} \int_{v}^{\infty}(\varsigma-v)^{\kappa-4}\left(\frac{1}{a(\varsigma)} \int_{\varsigma}^{\infty} q(s) \mathrm{d} s\right)^{1 / \beta} \mathrm{d} \varsigma=0,
\end{gathered}
$$

and

$$
\left(a(v)\left(y^{\prime}(v)\right)^{\beta}\right)^{\prime}+y^{\beta}(v) k q(v)\left(\frac{\zeta(\eta(v))}{\zeta(v)}\right)^{\beta}\left(\frac{\theta_{1}}{(\kappa-2) !} \eta^{\kappa-2}(v)\right)^{\beta}=0, \quad \theta_{1} \in(0,1)
$$

are oscillatory for every constant $\theta, \theta_{1} \in(0,1)$, then every solution of (1) is either oscillatory or satisfies $\lim _{v \rightarrow \infty} y(v)=0$.

Proof. Assume to the contrary that $y$ is a positive solution of (1). Then, we can suppose that $y(v)$ and $y(\eta(v))$ are positive for all $v \geq v_{1}$ sufficiently large. From Lemma 4 , we have three possible cases $\left(\mathbf{S}_{1}\right),\left(\mathbf{S}_{2}\right)$ and $\left(\mathbf{S}_{3}\right)$. Let case $\left(\mathbf{S}_{1}\right)$ hold. Using Lemma 2, we find

$$
y^{\prime}(v) \geq \frac{\theta}{(\kappa-2) !} v^{\kappa-2} y^{(\kappa-1)}(v),
$$


for every $\theta \in(0,1)$ and for all large $v$. We set

$$
\varphi(v):=\tau(v)\left(\frac{a(v)\left(y^{(\kappa-1)}(v)\right)^{\beta}}{y^{\beta}(v)}\right)
$$

and observe that $\varphi(v)>0$ for $v \geq v_{1}$, where $\tau \in C^{1}\left(\left[v_{0}, \infty\right),(0, \infty)\right)$ and

$$
\begin{aligned}
\varphi^{\prime}(v)= & \tau^{\prime}(v) \frac{a(v)\left(y^{(\kappa-1)}(v)\right)^{\beta}}{y^{\beta}(v)}+\tau(v) \frac{\left(a\left(y^{(\kappa-1)}\right)^{\beta}\right)^{\prime}(v)}{y^{\beta}(v)} \\
& -\beta \tau(v) \frac{y^{\beta-1}(v) y^{\prime}(v) a(v)\left(y^{(\kappa-1)}(v)\right)^{\beta}}{y^{2 \beta}(v)} .
\end{aligned}
$$

Using (12) and (13), we obtain

$$
\begin{aligned}
\varphi^{\prime}(v) \leq & \frac{\tau_{+}^{\prime}(v)}{\tau(v)} \varphi(v)+\tau(v) \frac{\left(a(v)\left(y^{(\kappa-1)}(v)\right)^{\beta}\right)^{\prime}}{y^{\beta}(v)} \\
& -\beta \tau(v) \frac{\theta}{(\kappa-2) !} v^{\kappa-2} \frac{a(v)\left(y^{(\kappa-1)}(v)\right)^{\beta+1}}{y^{\beta+1}(v)} \\
\leq & \frac{\tau^{\prime}(v)}{\tau(v)} \varphi(v)+\tau(v) \frac{\left(a(v)\left(y^{(\kappa-1)}(v)\right)^{\beta}\right)^{\prime}}{y^{\beta}(v)} \\
& -\frac{\beta \theta v^{\kappa-2}}{(\kappa-2) !(\tau(v) a(v))^{\frac{1}{\beta}}} \varphi(v)^{\frac{\beta+1}{\beta}} .
\end{aligned}
$$

From (1) and (14), we obtain

$$
\varphi^{\prime}(v) \leq \frac{\tau^{\prime}(v)}{\tau(v)} \varphi(v)-k \tau(v) \frac{q(v) y^{\beta}(\eta(v))}{y^{\beta}(v)}-\frac{\beta \theta v^{\kappa-2}}{(\kappa-2) !(\tau(v) a(v))^{\frac{1}{\beta}}} \varphi(v)^{\frac{\beta+1}{\beta}} .
$$

Note that $y^{\prime}(v)>0$ and $\eta(v) \geq v$, thus, we find

$$
\varphi^{\prime}(v) \leq \frac{\tau^{\prime}(v)}{\tau(v)} \varphi(v)-k \tau(v) q(v)-\frac{\beta \theta v^{\kappa-2}}{(\kappa-2) !(\tau(v) a(v))^{\frac{1}{\beta}}} \varphi(v)^{\frac{\beta+1}{\beta}} .
$$

If we set $\tau(v)=k=1$ in (15), then we find

$$
\varphi^{\prime}(v)+\frac{\beta \theta v^{\kappa-2}}{(\kappa-2) ! a^{\frac{1}{\beta}}(v)} \varphi(v)^{\frac{\beta+1}{\beta}}+q(v) \leq 0 .
$$

From [37], we can see that Equation (9) is non-oscillatory, which is a contradiction.

Let case $\left(\mathbf{S}_{2}\right)$ hold. If we set

$$
\psi(v):=\vartheta(v) \frac{y^{\prime}(v)}{y(v)}
$$

we see that $\psi(v)>0$ for $v \geq v_{1}$, where $\vartheta \in C^{1}\left(\left[v_{0}, \infty\right),(0, \infty)\right)$. By differentiating $\psi(v)$, we find

$$
\psi^{\prime}(v)=\frac{\vartheta^{\prime}(v)}{\vartheta(v)} \psi(v)+\vartheta(v) \frac{y^{\prime \prime}(v)}{y(v)}-\frac{1}{\vartheta(v)} \psi(v)^{2} .
$$


Now, by integrating (1) from $v$ to $m$ and using $y^{\prime}(v)>0$, we get

$$
a(m)\left(y^{(\kappa-1)}(m)\right)^{\beta}-a(v)\left(y^{(\kappa-1)}(v)\right)^{\beta}=-\int_{v}^{m} q(s) g(y(\eta(s))) d s .
$$

By virtue of $y^{\prime}(v)>0$ and $\eta(v) \geq v$, we get

$$
a(m)\left(y^{(\kappa-1)}(m)\right)^{\beta}-a(v)\left(y^{(\kappa-1)}(v)\right)^{\beta} \leq-k y^{\beta}(v) \int_{v}^{u} q(s) d s .
$$

Letting $m \rightarrow \infty$, we see that

$$
a(v)\left(y^{(\kappa-1)}(v)\right)^{\beta} \geq k y^{\beta}(v) \int_{v}^{\infty} q(s) \mathrm{d} s
$$

and so

$$
y^{(\kappa-1)}(v) \geq y(v)\left(\frac{k}{a(v)} \int_{v}^{\infty} q(s) \mathrm{d} s\right)^{1 / \beta} .
$$

Integrating again from $v$ to $\infty, \kappa-4$ times, we get

$$
y^{\prime \prime}(v)+\frac{y(v)}{(\kappa-4) !} \int_{v}^{\infty}(\varsigma-v)^{\kappa-4}\left(\frac{k}{a(\varsigma)} \int_{\varsigma}^{\infty} q(s) \mathrm{d} s\right)^{1 / \beta} \mathrm{d} \varsigma \leq 0 .
$$

From (16) and (17), we obtain

$$
\psi^{\prime}(v) \leq \frac{\vartheta^{\prime}(v)}{\vartheta(v)} \psi(v)-\frac{\vartheta(v)}{(\kappa-4) !} \mathfrak{\omega}(s)-\frac{1}{\vartheta(v)} \psi(v)^{2},
$$

where

$$
\omega(s)=\int_{v}^{\infty}(\varsigma-v)^{\kappa-4}\left(\frac{k}{a(\zeta)} \int_{\zeta}^{\infty} q(s) \mathrm{d} s\right)^{1 / \beta} \mathrm{d} \zeta .
$$

If we now set $\vartheta(v)=k=1$ in (18), then we obtain

$$
\psi^{\prime}(v)+\psi^{2}(v)+\frac{1}{(\kappa-4) !} \mathfrak{O}(s) \varsigma \leq 0 .
$$

From [37], we see Equation (10) is non-oscillatory, which is a contradiction.

Let case $\left(\mathbf{S}_{3}\right)$ hold. By recalling that $a(v)\left(y^{(\kappa-1)}(v)\right)^{\beta}$ is non-increasing, we obtain

$$
a^{1 / \beta}(s) y^{(\kappa-1)}(s) \leq a^{1 / \beta}(v) y^{(\kappa-1)}(v), s \geq v \geq v_{1} .
$$
we get

Dividing the latter inequality by $a^{1 / \beta}(s)$ and integrating the resulting inequality from $v$ to $u$,

$$
y^{(\kappa-2)}(u) \leq y^{(\kappa-2)}(v)+a^{1 / \beta}(v) y^{(\kappa-1)}(v) \int_{v}^{u} a^{-1 / \beta}(s) \mathrm{ds} .
$$

Letting $u \rightarrow \infty$, we obtain

$$
0 \leq y^{(\kappa-2)}(v)+a^{1 / \beta}(v) y^{(\kappa-1)}(v) \zeta(v) .
$$

Thus,

$$
\frac{-a^{1 / \beta}(v) y^{(\kappa-1)}(v) \zeta(v)}{y^{(\kappa-2)}(v)} \leq 1
$$


Furthermore, we get

$$
\left(\frac{y^{(\kappa-2)}(v)}{\zeta(v)}\right)^{\prime} \geq 0
$$

due to (19). Now define

$$
\phi(v)=\frac{a(v)\left(y^{(\kappa-1)}(v)\right)^{\beta}}{\left(y^{(\kappa-2)}(v)\right)^{\beta}}
$$

we see that $\phi(v)<0$ for $v \geq v_{1}$, and

$$
\phi^{\prime}(v)=\frac{\left(a(v)\left(y^{(\kappa-1)}(v)\right)^{\beta}\right)^{\prime}}{\left(y^{(\kappa-2)}(v)\right)^{\beta}}-\frac{\beta a(v)\left(y^{(\kappa-1)}(v)\right)^{\beta+1}}{\left(y^{(\kappa-2)}(v)\right)^{\beta+1}} .
$$

It follows from (1) and (19) that

$$
\phi^{\prime}(v)=\frac{-k q(v) y^{\beta}(\eta(v))}{\left(y^{(\kappa-2)}(v)\right)^{\beta}}-\frac{\beta \phi^{\beta / \beta+1}(v)}{a^{1 / \beta}(v)} .
$$

From Lemma 2, we find

$$
y(v) \geq \frac{\theta_{1}}{(\kappa-2) !} v^{\kappa-2} y^{(\kappa-2)}(v)
$$

Thus, we have

$$
\phi^{\prime}(v)=\frac{-k q(v) y^{\beta}(\eta(v))}{\left(y^{(\kappa-2)}(\eta(v))\right)^{\beta}} \frac{\left(y^{(\kappa-2)}(\eta(v))\right)^{\beta}}{\left(y^{(\kappa-2)}(v)\right)^{\beta}}-\frac{\beta \phi^{\beta / \beta+1}(v)}{a^{1 / \beta}(v)} .
$$

From (22), we obtain

$$
\phi^{\prime}(v) \leq-k q(v)\left(\frac{\theta_{1} \eta^{\kappa-2}(v)}{(\kappa-2) !}\right)^{\beta}\left(\frac{\zeta(\eta(v))}{\zeta(v)}\right)^{\beta}-\frac{\beta \phi^{\beta / \beta+1}(v)}{a^{1 / \beta}(v)} .
$$

From [37], we can see that Equation (11) is non-oscillatory, which is a contradiction.

Theorem 1 is proved.

Remark 1. It is well known (see [15]) that if

$$
\int_{v_{0}}^{\infty} \frac{1}{a(v)} \mathrm{d} v<\infty, \text { and } \liminf _{v \rightarrow \infty}\left(\int_{v_{0}}^{v} \frac{1}{a(s)} \mathrm{d} s\right)^{-1} \int_{v}^{\infty}\left(\int_{v_{0}}^{v} \frac{1}{a(s)} \mathrm{d} s\right)^{2} q(s) \mathrm{d} s>\frac{1}{4}
$$

then Equations (9)-(11) with $\beta=1$ are oscillatory.

Based on the above results and Theorem 1, we can easily obtain the following Hille and Nehari type oscillation criteria for (1) with $\beta=1$.

Theorem 2. Let $\beta=k=1$ and assume that (2) holds. If for $\theta, \theta_{1} \in(0,1)$

$$
\liminf _{v \rightarrow \infty}\left(\int_{v_{0}}^{v} \frac{\theta s^{\kappa-2}}{(\kappa-2) ! a(s)} \mathrm{d} s\right)^{-1} \int_{v}^{\infty}\left(\int_{v_{0}}^{v} \frac{\theta s^{\kappa-2}}{(\kappa-2) ! a(s)} \mathrm{d} s\right)^{2} q(s) \mathrm{d} s>\frac{1}{4},
$$

with

$$
\int_{v_{0}}^{\infty} \frac{\theta v^{\kappa-2}}{(\kappa-2) ! a(v)} \mathrm{d} v<\infty
$$


and if

$$
\begin{gathered}
\liminf _{v \rightarrow \infty} \int_{v_{0}}^{v} \frac{1}{(\kappa-4) !} \int_{v}^{v}(\varsigma-v)^{\kappa-4}\left(\frac{1}{a(\varsigma)} \int_{\varsigma}^{v} q(s) \mathrm{d} s\right)^{1 / \beta} \mathrm{d} \varsigma \mathrm{d} v>\frac{1}{4^{\prime}} \\
\liminf _{v \rightarrow \infty}\left(\int_{v_{0}}^{v} \frac{1}{a(s)} \mathrm{d} s\right)^{-1} \int_{v}^{\infty}\left(\int_{v_{0}}^{v} \frac{1}{a(s)} \mathrm{d} s\right)^{2} \frac{\theta_{1} \zeta(\eta(s)) \eta^{\kappa-2}(s) q(s)}{\zeta(s)(\kappa-2) !} \mathrm{d} s>\frac{1}{4},
\end{gathered}
$$

then every solution of (1) is either oscillatory or satisfies $\lim _{v \rightarrow \infty} y(v)=0$.

In the next theorem, we employ the integral averaging technique to establish a Philos-type oscillation criteria for (1):

Theorem 3. Let (2) holds. If there exist positive functions $\tau, \vartheta \in C^{1}\left(\left[v_{0}, \infty\right), \mathbb{R}\right)$ such that

$$
\begin{gathered}
\limsup _{v \rightarrow \infty} \frac{1}{H_{1}\left(v, v_{1}\right)} \int_{v_{1}}^{v}\left(H_{1}(v, s) k \tau(s) q(s)-\pi(s)\right) \mathrm{d} s=\infty, \\
\limsup _{v \rightarrow \infty} \frac{1}{H_{2}\left(v, v_{1}\right)} \int_{v_{1}}^{v}\left(H_{2}(v, s) \frac{\vartheta(s)}{(\kappa-4) !} \omega(s)-\frac{\vartheta(s) h_{2}^{2}(v, s)}{4}\right) \mathrm{d} s=\infty,
\end{gathered}
$$

and,

$$
\limsup _{v \rightarrow \infty} \frac{1}{H_{3}\left(v, v_{1}\right)} \int_{v_{1}}^{v}\left(H_{3}(v, s) k q(s)\left(\frac{\theta_{1} \eta^{\kappa-2}(s)}{(\kappa-2) !}\right)^{\beta} \zeta^{\beta}(\eta(s))-\tilde{\pi}(s)\right) \mathrm{d} s=\infty,
$$

where

$$
\pi(s)=\frac{h_{1}^{\beta+1}(v, s) H_{1}^{\beta}(v, s)}{(\beta+1)^{\beta+1}} \frac{((\kappa-2) !)^{\beta} \tau(s) a(s)}{\left(\theta s^{\kappa-2}\right)^{\beta}}
$$

and

$$
\tilde{\pi}(s)=\frac{\beta^{\beta+1} H_{3}(v, s)}{(\beta+1)^{\beta+1}} \frac{1}{a^{1 / \beta}(s) \zeta(s)} .
$$

Then every solution of (1) is either oscillatory or satisfies $\lim _{v \rightarrow \infty} y(v)=0$.

Proof. Assume to the contrary that $y$ is a positive solution of (1). Then, we can suppose that $y(v)$ and $y(\eta(v))$ are positive for all $v \geq v_{1}$ sufficiently large. From Lemma 4 , we have three possible cases $\left(\mathbf{S}_{1}\right),\left(\mathbf{S}_{2}\right)$ and $\left(\mathbf{S}_{3}\right)$. Assume that $\left(\mathbf{S}_{1}\right)$ holds. From Theorem 1, we get that (15) holds. Multiplying (15) by $H_{1}(v, s)$ and integrating the resulting inequality from $v_{1}$ to $v$ we find that

$$
\begin{aligned}
\int_{v_{1}}^{v} H_{1}(v, s) k \tau(s) q(s) \mathrm{d} s \leq & \varphi\left(v_{1}\right) H_{1}\left(v, v_{1}\right)+\int_{v_{1}}^{v}\left(\frac{\partial}{\partial s} H_{1}(v, s)+\frac{\tau^{\prime}(s)}{\tau(s)} H_{1}(v, s)\right) \varphi(s) \mathrm{d} s \\
& -\int_{v_{1}}^{v} \frac{\beta \theta s^{\kappa-2}}{(\kappa-2) !(\tau(s) a(s))^{\frac{1}{\beta}}} H_{1}(v, s) \varphi^{\frac{\beta+1}{\beta}}(s) \mathrm{d} s .
\end{aligned}
$$

From (3), we get

$$
\begin{aligned}
\int_{v_{1}}^{v} H_{1}(v, s) k \tau(s) q(s) \mathrm{d} s \leq & \varphi\left(v_{1}\right) H_{1}\left(v, v_{1}\right)+\int_{v_{1}}^{v} h_{1}(v, s) H_{1}^{\beta /(\beta+1)}(v, s) \varphi(s) \mathrm{d} s \\
& -\int_{v_{1}}^{v} \frac{\beta \theta s^{\kappa-2}}{(\kappa-2) !(\tau(s) a(s))^{\frac{1}{\beta}}} H_{1}(v, s) \varphi^{\frac{\beta+1}{\beta}}(s) \mathrm{d} s .
\end{aligned}
$$

Using Lemma 3 with $V=\beta \theta s^{\kappa-2} /\left((\kappa-2) !(\tau(s) a(s))^{\frac{1}{\beta}}\right) H_{1}(v, s), U=h_{1}(v, s) H_{1}^{\beta /(\beta+1)}(v, s)$ 
And $y=\varphi(s)$, we get

$$
\begin{aligned}
& h_{1}(v, s) H_{1}^{\beta /(\beta+1)}(v, s) \varphi(s)-\frac{\beta \theta s^{\kappa-2}}{(\kappa-2) !(\tau(s) a(s))^{\frac{1}{\beta}}} H_{1}(v, s) \varphi^{\frac{\beta+1}{\beta}}(s) \\
\leq & \frac{h_{1}^{\beta+1}(v, s) H_{1}^{\beta}(v, s)}{(\beta+1)^{\beta+1}} \frac{((\kappa-2) !)^{\beta} \tau(s) a(s)}{\left(\theta s^{\kappa-2}\right)^{\beta}},
\end{aligned}
$$

which, with (29) gives

$$
\frac{1}{H_{1}\left(v, v_{1}\right)} \int_{v_{1}}^{v}\left(H_{1}(v, s) k \tau(s) q(s)-\pi(s)\right) \mathrm{d} s \leq \varphi\left(v_{1}\right),
$$

which contradicts (27). Assume that $\left(\mathbf{S}_{2}\right)$ holds. From Theorem 1, we get that (18) holds. Multiplying (18) by $H_{2}(v, s)$ and integrating the resulting inequality from $v_{1}$ to $v$, we obtain

$$
\begin{aligned}
\int_{v_{1}}^{v} H_{2}(v, s) \frac{\vartheta(s)}{(\kappa-4) !} \mathscr{}(s) \mathrm{d} s \leq & \psi\left(v_{1}\right) H_{2}\left(v, v_{1}\right) \\
& +\int_{v_{1}}^{v}\left(\frac{\partial}{\partial s} H_{2}(v, s)+\frac{\vartheta^{\prime}(s)}{\vartheta(s)} H_{2}(v, s)\right) \psi(s) \mathrm{d} s \\
& -\int_{v_{1}}^{v} \frac{1}{\vartheta(s)} H_{2}(v, s) \psi^{2}(s) \mathrm{d} s .
\end{aligned}
$$

Thus, from (4), we obtain

$$
\begin{aligned}
\int_{v_{1}}^{v} H_{2}(v, s) \frac{\vartheta(s)}{(\kappa-4) !} \mathscr{Q}(s) \mathrm{d} s \leq & \psi\left(v_{1}\right) H_{2}\left(v, v_{1}\right)+\int_{v_{1}}^{v} h_{2}(v, s) \sqrt{H_{2}(v, s)} \psi(s) \mathrm{d} s \\
& -\int_{v_{1}}^{v} \frac{1}{\vartheta(s)} H_{2}(v, s) \psi^{2}(s) \mathrm{d} s \\
\leq & \psi\left(v_{1}\right) H_{2}\left(v, v_{1}\right)+\int_{v_{1}}^{v} \frac{\vartheta(s) h_{2}^{2}(v, s)}{4} \mathrm{~d} s
\end{aligned}
$$

and so

$$
\frac{1}{H_{2}\left(v, v_{1}\right)} \int_{v_{1}}^{v}\left(H_{2}(v, s) \frac{\vartheta(s)}{(\kappa-4) !} \omega(s)-\frac{\vartheta(s) h_{2}^{2}(v, s)}{4}\right) \mathrm{d} s \leq \psi\left(v_{1}\right),
$$

which contradicts (28). Assume that $\left(\mathbf{S}_{3}\right)$ holds. Using (19) and (21), we see that

$$
-\phi(v) \zeta^{\beta}(v) \leq 1
$$

due to (30). Multiplying this inequality by $\zeta^{\beta}(v)$ and integrating the resulting inequality from $v_{1}$ to $v$, we get

$$
\begin{gathered}
\zeta^{\beta}(v) \phi(v)-\zeta^{\beta}\left(v_{1}\right) \phi\left(v_{1}\right)+\beta \int_{v_{1}}^{v} a^{-1 / \beta}(s) \zeta^{\beta-1}(s) \phi(s) d s \\
\leq-\int_{v_{1}}^{v} k q(s)\left(\frac{\theta_{1} \eta^{\kappa-2}(s)}{(\kappa-2) !}\right)^{\beta} \zeta^{\beta}(\eta(s)) d s-\beta \int_{v_{1}}^{v} \frac{\phi^{\beta / \beta+1}(s)}{a^{1 / \beta}(s)} \zeta^{\beta}(s) d s .
\end{gathered}
$$


Multiplying (31) by $H_{3}(v, s)$, we find that

$$
\begin{aligned}
\int_{v_{1}}^{v} H_{3}(v, s) k q(s)\left(\frac{\theta_{1} \eta^{\kappa-2}(s)}{(\kappa-2) !}\right)^{\beta} \zeta^{\beta}(\eta(s)) \mathrm{d} s \leq & \zeta^{\beta}\left(v_{1}\right) \phi\left(v_{1}\right) H_{3}\left(v, v_{1}\right)-\zeta^{\beta}(v) \phi(v) H_{3}\left(v, v_{1}\right) \\
& +\int_{v_{1}}^{v} \beta a^{-1 / \beta}(s) \zeta^{\beta-1}(s) \phi(s) H_{3}(v, s) \mathrm{d} s \\
& -\int_{v_{1}}^{v} \frac{\beta \phi^{\beta / \beta+1}(s)}{a^{1 / \beta}(s)} \zeta^{\beta}(s) H_{3}(v, s) \mathrm{d} s .
\end{aligned}
$$
we get

Using Lemma 3 with $V=\zeta^{\beta}(s) H_{3}(v, s) / a^{1 / \beta}(s), U=a^{-1 / \beta}(s) \zeta^{\beta-1}(s) H_{3}(v, s)$ and $y=\phi(s)$,

$$
\begin{aligned}
& \beta a^{-1 / \beta}(s) \zeta^{\beta-1}(s) \phi(s) H_{3}(v, s)-\frac{\beta \phi^{\beta / \beta+1}(s)}{a^{1 / \beta}(s)} \zeta^{\beta}(s) H_{3}(v, s) \\
\leq & \frac{\beta^{\beta+1} H_{3}(v, s)}{(\beta+1)^{\beta+1}} \frac{1}{a^{1 / \beta}(s) \zeta(s)}
\end{aligned}
$$

and easily, we find that

$$
\frac{1}{H_{3}\left(v, v_{1}\right)} \int_{v_{1}}^{v}\left(H_{3}(v, s) k q(s)\left(\frac{\theta_{1} \eta^{\kappa-2}(s)}{(\kappa-2) !}\right)^{\beta} \zeta^{\beta}(\eta(s))-\tilde{\pi}(s)\right) \mathrm{d} s \leq \zeta^{\beta}\left(v_{1}\right) \phi\left(v_{1}\right)+1,
$$

which contradicts (27). This completes the proof.

Example 1. We consider the equation

$$
\left(v^{5} y^{\prime \prime \prime}(v)\right)^{\prime}+v q_{0} y(3 v)=0, v \geq 1
$$

where $q_{0}>0$ is a constant. Note that $\beta=1, \kappa=4, a(v)=v^{5}, q(v)=v q_{0}$ and $\eta(v)=3 v$. If we set $k=1$, then condition (24) becomes

$$
\begin{aligned}
& \liminf _{v \rightarrow \infty}\left(\int_{v_{0}}^{v} \frac{\theta s^{\kappa-2}}{(\kappa-2) ! a(s)} \mathrm{d} s\right)^{-1} \int_{v}^{\infty}\left(\int_{v_{0}}^{v} \frac{\theta s^{\kappa-2}}{(\kappa-2) ! a(s)} \mathrm{d} s\right)^{2} q(s) \mathrm{d} s \\
= & \liminf _{v \rightarrow \infty}\left(4 v^{2}\right) \int_{v}^{\infty} \frac{q_{0}}{16 s^{3}} d s=\liminf _{v \rightarrow \infty}\left(4 v^{2}\right)\left(\frac{q_{0}}{32 v^{2}}\right) \\
= & \frac{q_{0}}{8}>\frac{1}{4}
\end{aligned}
$$

while condition (25) becomes

$$
\begin{aligned}
\liminf _{v \rightarrow \infty} v \int_{v_{0}}^{v} \frac{1}{(\kappa-4) !} \int_{v}^{v}(\varsigma-v)^{\kappa-4}\left(\frac{1}{a(\varsigma)} \int_{\varsigma}^{v} q(s) \mathrm{d} s\right)^{1 / \beta} \mathrm{d} \varsigma \mathrm{d} v & =\liminf _{v \rightarrow \infty} v\left(\frac{q_{0}}{4 v}\right) \\
& =\frac{q_{0}}{4}>\frac{1}{4},
\end{aligned}
$$

and hence condition (26) is satisfied. Therefore, from Theorem 2, all solutions of Equation (32) are oscillatory if $q_{0}>2$.

Remark 2. One can easily see that the results obtained in $[18,19]$ cannot be applied to conditions in Theorem 2, so our results are new. 
Remark 3. We can generalize our results by studying the equation in the form

$$
\left(a(v)\left(y^{(\kappa-1)}(v)\right)^{\beta}\right)^{\prime}+\sum_{i=1}^{j} q_{i}(v) y^{\beta}\left(\eta_{i}(v)\right)=0, \text { where } v \geq v_{0}, j \geq 1
$$

For this we leave the results to researchers interested.

\section{Conclusions}

In this article we studied we provided three new Theorems on the oscillatory and asymptotic behavior of a class of even-order advanced differential equations with a non-canonical operator in the form of (1).

For researchers interested in this field, and as part of our future research, there is a nice open problem which is finding new results in the following cases:

$$
\begin{aligned}
\left(\mathbf{S}_{1}\right) \quad & y(v)>0, y^{\prime}(v)>0, y^{(\kappa-2)}(v)>0, y^{(\kappa-1)}(v) \leq 0, \quad\left(a(v)\left(y^{(\kappa-1)}(v)\right)^{\beta}\right)^{\prime} \leq 0, \\
\left(\mathbf{S}_{2}\right) \quad & y(v)>0, y^{(r)}(v)<0, y^{(r+1)}(v)>0, \forall r \in\{1,3, \ldots, \kappa-3\}, \\
& \text { and } y^{(\kappa-1)}(v)<0,\left(a(v)\left(y^{(\kappa-1)}(v)\right)^{\beta}\right)^{\prime} \leq 0 .
\end{aligned}
$$

For all this there is some research in progress.

Author Contributions: The authors claim to have contributed equally and significantly in this paper. All authors have read and agreed to the published version of the manuscript.

Funding: This work is supported by Science Foundation Ireland (SFI), by funding Ioannis Dassios, under Investigator Programme Grant No. SFI/15 /IA/3074.

Acknowledgments: The authors thank the reviewers for for their useful comments, which led to the improvement of the content of the paper.

Conflicts of Interest: There are no competing interests between the authors.

\section{References}

1. Bazighifan, O.; Postolache, M. An improved conditions for oscillation of functional nonlinear differential equations. Mathematics 2020, 8, 552. [CrossRef]

2. Bazighifan, O.; Elabbasy, E.M.; Moaaz, O. Oscillation of higher-order differential equations with distributed delay. J. Inequal.Appl. 2019, 55, 1-9.

3. Bazighifan, O. An Approach for Studying Asymptotic Properties of Solutions of Neutral Differential Equations. Symmetry 2020, 12, 555. [CrossRef]

4. Cesarano, C.; Pinelas, S.; Al-Showaikh, F.; Bazighifan, O. Asymptotic Properties of Solutions of Fourth-Order Delay Differential Equations. Symmetry 2019, 11, 628. [CrossRef]

5. Bazighifan, O.; Cesarano, C. A Philos-Type Oscillation Criteria for Fourth-Order Neutral Differential Equations. Symmetry 2020, 12, 379. [CrossRef]

6. Cesarano, C.; Bazighifan, O. Qualitative behavior of solutions of second order differential equations. Symmetry 2019, 11, 777. [CrossRef]

7. Grace, S.; Dzurina, J.; Jadlovska, I.; Li, T. On the oscillation of fourth order delay differential equations. Adv. Differ.Equ. 2019, 118, 1-15. [CrossRef]

8. Gyori, I.; Ladas, G. Oscillation Theory of Delay Differential Equations with Applications; Clarendon Press: Oxford, UK, 1991.

9. Li, T.; Baculikova, B.; Dzurina, J.; Zhang, C. Oscillation of fourth order neutral differential equations with p-Laplacian like operators. Bound. Value Probl. 2014, 56, 41-58. [CrossRef]

10. Moaaz, O.; Elabbasy, E.M.; Bazighifan, O. On the asymptotic behavior of fourth-order functional differential equations. Adv. Differ. Equ. 2017, 261, 1-13. [CrossRef]

11. Moaaz, O.; Dassios, I.; Bazighifan, O.; Muhib, A. Oscillation Theorems for Nonlinear Differential Equations of Fourth-Order. Mathematics 2020, 8, 520. [CrossRef] 
12. Bazighifan, O.; Dassios, I. Riccati Technique and Asymptotic Behavior of Fourth-Order Advanced Differential Equations. Mathematics 2020, 8, 590. [CrossRef]

13. Moaaz, O.; Dassios, I.; Bazighifan, O. Oscillation Criteria of Higher-order Neutral Differential Equations with Several Deviating Arguments. Mathematics 2020, 8, 402. [CrossRef]

14. Moaaz, O.; Muhib, A. New oscillation criteria for nonlinear delay differential equations of fourth-order. Appl. Math.Comput. 2020, 377, 125192. [CrossRef]

15. Nehari, Z. Oscillation criteria for second order linear differential equations. Trans. Am. Math. Soc. 1957, 85, 428-445. [CrossRef]

16. Philos, C. On the existence of nonoscillatory solutions tending to zero at $\infty$ for differential equations with positive delay. Arch. Math. 1981, 36, 168-178. [CrossRef]

17. Rehak, P. How the constants in Hille-Nehari theorems depend on time scales. Adv. Differ. Equ. 2006, 2006, 064534. [CrossRef]

18. Zhang, C.; Agarwal, R.P.; Bohner, M.; Li, T. New results for oscillatory behavior of even-order half-linear delay differential equations. Appl. Math. Lett. 2013, 26, 179-183. [CrossRef]

19. Moaaz, O.; Kumam, P.; Bazighifan, O. On the Oscillatory Behavior of a Class of Fourth-Order Nonlinear Differential Equation. Symmetry 2020, 12, 524. [CrossRef]

20. Zhang, C.; Li, T.; Saker, S. Oscillation of fourth-order delay differential equations. J. Math. Sci. 2014, 201, $296-308$. [CrossRef]

21. Tzounas, G.; Dassios, I.; Milano, F. Modal Participation Factors of Algebraic Variables. IEEE Trans. Power Syst. 2020, 35, 742-750. [CrossRef]

22. Dassios, I.; Tzounas, G.; Milano, F. Generalized fractional controller for singular systems of differential equations. J. Comput. Appl. Math. 2020, 378, 112919. [CrossRef]

23. Dassios, I.; Tzounas, G.; Milano, F. Participation Factors for Singular Systems of Differential Equations Circuits. Syst. Signal Process.2020, 39, 83-110. . [CrossRef]

24. Dassios, I.; Tzounas, G.; Milano, F. The Mobius transform effect in singular systems of differential equations. Appl. Math. Comput. 2019, 361, 338-353. [CrossRef]

25. Liu, M.; Dassios, I.; Tzounas, G.; Milano, F. Model-Independent Derivative Control Delay Compensation Methods for Power Systems. Energies 2020, 13, 342. [CrossRef]

26. Liu, M.; Dassios, I.; Tzounas, G.; Milano, F. Stability Analysis of Power Systems with Inclusion of Realistic-Modeling of WAMS Delays. IEEE Trans. Power Syst. 2019, 34, 627-636. [CrossRef]

27. Dassios, I. Stability of Bounded Dynamical Networks with Symmetry. Symmetry 2018, 10, 121. [CrossRef]

28. Boutarfa, B.; Dassios, I. A stability result for a network of two triple junctions on the plane. Math. Methods Appl. Sci. 2017, 40, 6076-6084. [CrossRef]

29. Dassios, I. Stability of basic steady states of networks in bounded domains. Comput. Math. Appl. 2015, 70, 2177-2196.

30. O'Keeffe, G.; Dassios, I. Ideas From Bounded Confidence Theory Applied to Dynamical Networks of Interacting Free-Bodies. Front. Phys. 2019, 7, 131. [CrossRef]

31. Dassios, I.; Baleanu, D. Optimal solutions for singular linear systems of Caputo fractional differential equations. Math. Methods Appl. Sci. 2020. [CrossRef]

32. Dassios, I.; Baleanu, D. Caputo and related fractional derivatives in singular systems. Appl. Math. Comput. 2018, 337, 591-606. [CrossRef]

33. Hale, J.K. Theory of Functional Differential Equations; Springer: New York, NY, USA, 1977.

34. Agarwal, R.; Grace, S.R. Oscillation theorems for certain functional differential equations of higher order. Math. Comput. Model. 2004, 39, 1185-1194. [CrossRef]

35. Agarwal, R.; Grace, S.R.; O'Regan, D. Oscillation criteria for certain $\mathrm{n}$ th order differential equations with deviating arguments. J. Math. Anal. Appl. 2001, 262, 601-622. [CrossRef] 
36. Grace, S.R.; Lalli, B.S. Oscillation theorems for nth-order differential equations with deviating arguments. Proc. Am. Math. Soc. 1984, 90, 65-70.

37. Agarwal, R.; Shieh, S.L.; Yeh, C.C. Oscillation criteria for second order retarded differential equations. Math. Comput. Model. 1997, 26,1-11. [CrossRef]

(C) 2020 by the authors. Licensee MDPI, Basel, Switzerland. This article is an open access article distributed under the terms and conditions of the Creative Commons Attribution (CC BY) license (http:/ / creativecommons.org/licenses/by/4.0/). 